\title{
A tricky sticky eye
}

Tim Staunton Smith

CASE

A man, 31 years of age, presented to a university medical centre on a Monday afternoon with a two-day history of a red right eye with mucopurulent discharge. There was no visual loss, eye discomfort or photophobia. He had no known contacts and no past history of eye problems.

Examination revealed right eye conjunctival redness but no tenderness over the eyelid or lacrimal sac and no florid discharge. Visual acuity was normal. A provisional diagnosis of conjunctivitis was made and the patient was commenced on a course of chloramphenicol eye drops, two drops four times per day.

In addition to his right eye symptoms, the patient requested a sexual health screen. He stated he was last in a relationship two months earlier with a male partner and had engaged in protected anal sex and unprotected oral sex. A pathology request form was generated for bloodborne viruses, together with chlamydia and gonorrhoea polymerase chain reaction (PCR) testing of a urine sample, anal swab and throat swab.

The patient returned to the medical centre and consulted a different general practitioner (GP) on Thursday of the same week. His right eye felt worse despite applying chloramphenicol drops regularly. On examination, visual acuity was again normal and an inflamed right conjunctiva was noted.

The reviewing GP read his colleague's consultation notes from earlier in the week, which acknowledged the yet-tobe-completed request for a sexual health screen, prompting the reviewing GP for further sexual history-taking. Despite having stated in his previous consultation that his last relationship was in January, the patient stated now that he had engaged in sexual activity five days prior to the onset of his right eye symptoms, during which his sexual partner's ejaculate contacted his right eye.

Swabs of the conjunctiva were collected for microscopy, culture and sensitivity (MCS) and PCR, and a $1 \mathrm{~g}$ single dose of azithromycin was prescribed. On review the following day, the patient's eye symptoms and signs were almost completely resolved. The conjunctival PCR swab was positive for chlamydia. Contact tracing was discussed with the patient and arrangements made for completion of his initially requested sexually transmissible infection (STI) screen.

\section{Question 1}

What are the aetiological agents of acute conjunctivitis?

\section{Question 2}

How often is chlamydia the aetiological agent in acute conjunctivitis? Is it notifiable?

\section{Question 3}

Are there any distinguishing clinical features that help differentiate chlamydia as the aetiological agent in acute conjunctivitis? What else helps accurately establish the diagnosis?

\section{Question 4}

Do current sexual health guidelines discuss conjunctivitis as a symptom of chlamydia? Do they recommend enquiring about eye exposure as part of sexual history-taking?

\section{Question 5}

Does this case represent a case of trachoma?

\section{Answer 1}

Acute conjunctivitis can be infective (eg viral, bacterial, chlamydial) or non- infective (eg allergies, irritants). ${ }^{1}$ Affected patients can be grouped into four age categories, reflecting different relative frequency of aetiological agents:

- neonates

- older infants and toddlers (aged $>1$ year)

- school-aged children (aged $>5$ years)

- adults. $^{2}$

\section{Answer 2}

Chlamydia is currently the most frequently reported STI in Australia, with 89,486 diagnoses in $2016 .{ }^{3}$ It is estimated to account for $1.8-5.6 \%$ of all cases of acute conjunctivitis. ${ }^{4}$ However, unlike urogenital infection, chlamydia conjunctivitis is not notifiable and is specifically excluded from chlamydia laboratory case definition. ${ }^{5}$ This, together with the usual self-limiting pattern of acute conjunctivitis, makes accurate prevalence rates for acute conjunctivitis caused by chlamydia difficult to ascertain.

\section{Answer 3}

The presence of mucopurulent discharge and follicular eyelid reaction may be helpful clinical features of chlamydia conjunctivitis. ${ }^{1,2,4}$ However, clinical presentation is often non-specific and reliance on signs and symptoms does not reliably lead to an accurate diagnosis in acute conjunctivitis. ${ }^{4}$ Given this ambiguity, establishing risk by enquiring about exposure to potentially infectious genital secretions through autoinoculation or sexual contact is perhaps the most important piece of information in differentiating chlamydia as the likely aetiological agent in acute conjunctivitis, particularly in light of the large numbers of reported genital chlamydia infections in Australia. ${ }^{3}$

\section{Answer 4}

The current Australian STI management guidelines do not list conjunctivitis as a potential symptom of chlamydia infection, nor do they recommend enquiring about 
genital secretion eye exposure as part of sexual history-taking. ${ }^{6}$ The guidelines do mention sight-threatening conjunctivitis caused by gonorrhoea ${ }^{6}$ and, with a threefold increase in gonorrhoea notifications over the past decade, ${ }^{3}$ it should be considered in the differential diagnosis of a mucopurulent conjunctivitis. Clinicians should take appropriate precautions to avoid contact with potentially infectious discharge in patients presenting with conjunctivitis.

\section{Answer 5}

No. Trachoma is a specific condition related to repeated chlamydia eye infection with non-urogenital strains and an accompanying inflammatory reaction, bacterial co-infection, anatomical changes and corneal scarring that is a significant cause of blindness in some remote Aboriginal and Torres Strait Islander communities. ${ }^{4}$ However, acute conjunctivitis can be caused by trachoma strains and urogenital strains of chlamydia, and is sometimes referred to as paratrachoma. ${ }^{7}$

\section{Key points}

- Chlamydia should be considered a potential aetiological agent in conjunctivitis in sexually active people, particularly given established high rates of urogenital chlamydia infection.

- Asking about possible eye exposure to genital secretions and/or eye symptoms should be considered when conducting a sexual health history.

- Reviewing consultation notes of colleagues can provide vital prompts in making an accurate diagnosis.

\section{Author}

Tim Staunton Smith MBBS, FRACGP, MPH, Senior Lecturer, Monash Rural Health, Latrobe Valley and West Gippsland, Traralgon West, Vic; General Practitioner, Swinburne University Health Service, Hawthorn, Vic. timothy.stauntonsmith@monash.edu Competing interests: None.

Provenance and peer review: Not commissioned, externally peer reviewed.

\section{References}

1. Cronau H, Kankanala RR, Mauger T. Diagnosis and management of red eye in primary care. Am Fam Physician 2010;81(2):137-44.

2. Thanathanee O, O'Brien TP. Conjunctivitis: Systematic approach to diagnosis and therapy. Curr Infect Dis Rep 2011;13(2):141-48. doi: 10.1007/ s11908-011-0167-y.

3. National Notifiable Diseases Surveillance System. Notifications of a selected disease by month and year, 1991 to present. Available at www9.health. gov.au/cda/source/rpt_3_sel.cfm [Accessed 10 April 2018].

4. Azari AA, Barney NP. Conjunctivitis: A systematic review of diagnosis and treatment. JAMA 2013;310(16):1721-29. doi: 10.1001/ jama.2013.280318.

5. Department of Health. Chlamydia laboratory case definition (LCD). Canberra: DoH, 2009. Available at www.health.gov.au/internet/main/ publishing.nsf/Content/cda-phlncd-chlamydia. htm [Accessed 28 November 2017].

6. Australasian Sexual Health Alliance. Australian STI management guidelines for use in primary care. Darlinghurst, NSW: ASHA, 2016. Available at www.sti.guidelines.org.au [Accessed 28 November 2017].

7. Giffard PM, Brenner NC, Tabrizi SN, et al. Chlamydia trachomatis genotypes in a crosssectional study of urogenital samples from remote Northern and Central Australia. BMJ Open 2016;6:e00964.

correspondence ajgp@racgp.org.au 